\title{
Aberration correction in energy loss spectrometers and monochromators
}

\author{
O.L. Krivanek, J.P. Ursin, G.J. Corbin, N. Dellby, M.F. Murfitt, C.S. Own and Z.S. Szilagyi
}

Nion Co., $11028^{\text {th }}$ St., Kirkland, WA 98033, USA

Early electron energy-loss spectrometers made sure that spectra were focused to first-order in the energy dispersion direction and sometimes also in the non-dispersion direction, but they did not worry about higher-order focusing. This was the case for the pioneering post-column spectrometer built by David Wittry during his sabbatical in Cambridge UK [1], which introduced important innovations such as a magnetic prism with a large entrance distance that enabled it to focus on the small crossover in the back-focal plane of the microscope's final projector, thereby allowing spectra to be recorded in all the regular operating modes of the microscope.

Second-order aberration correction for spectrometers used in electron microscopes was introduced by Crewe et al. [2]. There are two principal second-order aberrations to correct [3]: $\mathrm{d}^{2} \mathrm{x} / \mathrm{dx}^{, 2}$ and $\mathrm{d}^{2} \mathrm{x} / \mathrm{dy}{ }^{2}$, i.e. the second-order focus in the energy dispersion direction $(=\mathrm{x})$ for electrons rays displaced in the $x$ direction in the entrance aperture of the spectrometer $\left(x^{\prime}=\mathrm{dx} / \mathrm{dz} \neq 0\right)$, and those displaced in the $y$ direction $\left(y^{\prime}=d y / d z \neq 0\right)$. The two terms can be corrected simultaneously by two sextupoles acting on the electron beam at locations in which the beam aspect ratios (width in $\mathrm{x}$ divided by width in y) are different. Curved entrance and exit faces of a magnetic prism produce sextupole moments and can thus be used for the correction too. Surprisingly, Crewe et al. designed a symmetric spectrometer in which the beam had the same aspect ratio at both the entrance and the exit of the prism. The curved entrance and exit faces of their prism therefore only corrected $\mathrm{d}^{2} \mathrm{x} / \mathrm{dx}{ }^{2}$, and they made $\mathrm{d}^{2} \mathrm{x} / \mathrm{dy}{ }^{2}$ two times worse than it would have been with an uncorrected prism.

Simultaneous correction of the two coefficients plus minimization of the tilt of the spectrum plane (a second-rank aberration described by $\mathrm{d}^{2} \mathrm{x} / \mathrm{dx}$ ' $\mathrm{dE}$ ) was accomplished by an asymmetric prism with entrance and exit curvatures of opposite signs [4-6]. Second-order correction was also introduced for in-column magnetic imaging filters [7]. In all these instances, use was made of the general principle that a series of sextupoles (or sextupole moments produced by curved prism faces), each one acting on a beam whose first-order properties are different from all the other sextupole locations, can correct several different second-order aberrations. The effect of each sextupole is of course not pure: it changes a mixture of second-order aberration terms. The mixture proportions are determined by the first-order properties of the beam inside the sextupole. A combination of sextupole excitations that together null all the chosen aberration coefficients does, however, usually exist.

Thorough use of the above principle was made in post-column imaging filters. For instance, the first Gatan imaging filter [8] used 6 sextuples in addition to curved entrance and exit faces of its magnetic prism, i.e. 8 second-order correcting elements in total. Correction of two second-order spectrum aberrations and six second-order image aberrations (or distortions) was achieved because the first-order properties of the beam in each sextupole were very different. The design maximized the differences in the beam properties, with the result that typically one or at most two sextupoles affected each aberration/distortion. This made setting the filter's correcting elements to their proper excitations much easier than if all 8 elements affected all the 8 aberrations/distortions nearly equally. 
Correcting aberrations by high-order optical elements acting on beams of different first-order properties is also the principle used for round-beam $\mathrm{C}_{\mathrm{s}}$ correction in quadrupole/octupole correctors: quadrupoles produce beams of different properties in at least three separate octupoles, the octupoles produce different mixtures of $\mathrm{C}_{3,0}, \mathrm{C}_{3,2}$ and $\mathrm{C}_{3,4}$ aberrations ( $=\mathrm{C}_{\mathrm{s}}$ plus 2-fold and 4-fold astigmatisms of $\mathrm{C}_{\mathrm{s}}$ ), and are set to give adjustable $\mathrm{C}_{3,0}$ and nulled $\mathrm{C}_{3,2}$ and $\mathrm{C}_{3,4}$. This is why $\mathrm{C}_{\mathrm{s}}$ correction seemed a very solvable problem to the Nion and probably also the Rose/CEOS design teams, both of which had much experience with second-order correction before producing practical third-order correctors.

Monochromators used in STEM occupy an interesting niche between spectrometers and round-beam correctors: inside the monochromator, only aberrations affecting the quality of the spectrum produced at the energy-selecting slit are of major concern, whereas at the exit of the monochromator, aberrations affecting the probe formation are key. We have recently designed a monochromator [9] which acts on both classes of aberrations and should be able to provide energy loss spectra of 10-30 meV energy resolution, and also correct the chromatic aberration of the probeforming column (Fig. 1). A major feature of the design is that it ties the energy analyzed by the energy-loss spectrometer and the high voltage of the electron microscope to the energy being selected by the monochromator. This improves spectrum energy stability compared to what is achievable with stable but separate power supplies, and makes sub 50-meV energy resolution feasible. Probe $\mathrm{C}_{\mathrm{c}}$ correction is accomplished by correcting both important second-rank geometricchromatic aberrations $\left(\mathrm{d}^{2} \mathrm{x} / \mathrm{dx}{ }^{\prime} \mathrm{dE}\right.$ and $\left.\mathrm{d}^{2} \mathrm{y} / \mathrm{dy} \mathrm{d}^{\prime} \mathrm{dE}\right)$ at the monochromator slit and at the exit crossover. It should improve the attainable spatial resolution of the STEM significantly, especially at primary voltages of $100 \mathrm{kV}$ and lower.

[1] D.B. Wittry, J. Phys. D: Appl. Phys. 2 (1969) 1757.

[2] A.V. Crewe, M. Isaacson and D. Johnson, Rev. Sci. Inst. 42 (1971) 411.

[3] R.F. Egerton (1996) "EELS in the Electron Microscope", 2nd ed., Plenum, New York.

[4] W. Engel, Proc. $9^{\text {th }}$ Int. EM Congr. (Toronto) 1 (1978) 48.

[5] H. Shuman, Ultramicroscopy 5 (1980) 45.

[6] M. Scheinfein and M. Isaacson, Scanning Electron Microscopy 4 (1984) 1681.

[7] H. Rose and D. Krahl (1995) in "Energy-Filtering Transmission Electron Microscopy", L.

Reimer, Ed., Springer, Heidelberg, p. 43.

[8] O.L. Krivanek, A.J. Gubbens and N. Dellby, Microsc., Microanal., Microstr. 2 (1991) 315.

[9] O.L. Krivanek et al., Phil Trans Roy Soc. (2009), in print; US patent application.

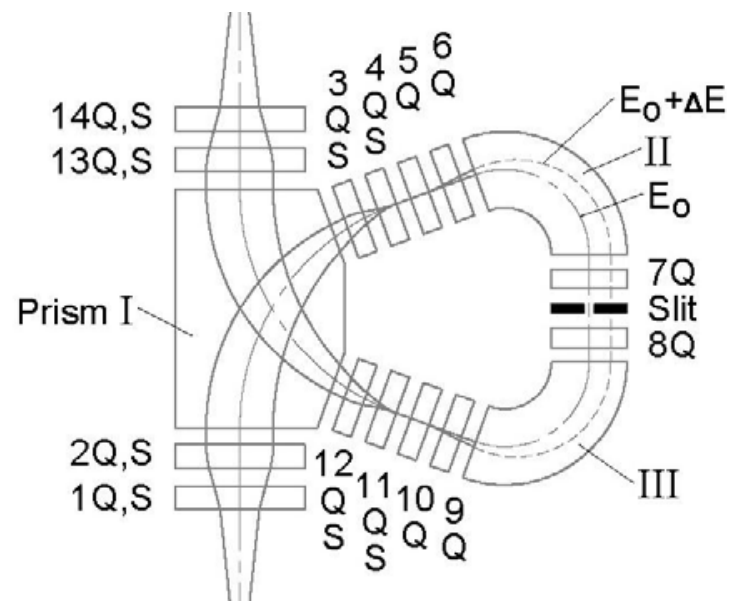

FIG. 1. The proposed monochromator. Q's denote quadrupoles, S's sextupoles, the numbers denote the optical element layers. Quadrupoles 3-6 allow the energy dispersion at the slit to be varied, quadrupoles 9-12 null the dispersion of the output beam. Sextupoles 1-4 and 11-14 correct all important second-order geometric and second-rank mixed geometric-chromatic aberrations. 